\title{
Circadian modulation of short-term memory in Drosophila
}

\author{
Lisa C. Lyons ${ }^{1-3}$ and Gregg Roman ${ }^{1}$ \\ ${ }^{1}$ Department of Biology and Biochemistry, University of Houston, Houston, Texas 77204, USA; ${ }^{2}$ Department of \\ Biological Science, Florida State University, Tallahassee, Florida 32306, USA
}

\begin{abstract}
Endogenous biological clocks are widespread regulators of behavior and physiology, allowing for a more efficient allocation of efforts and resources over the course of a day. The extent that different processes are regulated by circadian oscillators, however, is not fully understood. We investigated the role of the circadian clock on short-term associative memory formation using a negatively reinforced olfactory-learning paradigm in Drosophila melanogaster. We found that memory formation was regulated in a circadian manner. The peak performance in short-term memory (STM) occurred during the early subjective night with a twofold performance amplitude after a single pairing of conditioned and unconditioned stimuli. This rhythm in memory is eliminated in both timeless and period mutants and is absent during constant light conditions. Circadian gating of sensory perception does not appear to underlie the rhythm in short-term memory as evidenced by the nonrhythmic shock avoidance and olfactory avoidance behaviors. Moreover, central brain oscillators appear to be responsible for the modulation as cryptochrome mutants, in which the antennal circadian oscillators are nonfunctional, demonstrate robust circadian rhythms in short-term memory. Together these data suggest that central, rather than peripheral, circadian oscillators modulate the formation of short-term associative memory and not the perception of the stimuli.
\end{abstract}

Circadian clocks operate in organisms ranging from single-celled prokaryotes to humans. Intracellular circadian oscillators maintain $24 \mathrm{~h}$ cycles through circadian gene expression and autoregulatory, negative feedback loops (reviewed in Bell-Pedersen et al. 2005; Hardin 2005). Circadian regulation impacts almost every aspect of an animal's life including gene expression, enzyme activity, cell division, cell metabolism, as well as physiological and behavioral processes. Given the widespread effect of the circadian clock on physiology and behavior, it is not surprising that the circadian clock also affects learning and memory. In the past several years, circadian modulation of long-term memory formation has been observed in many invertebrate and vertebrate model systems including Aplysia (Fernandez et al. 2003; Lyons et al. 2005), zebrafish (Rawashdeh et al. 2007), mice (Valentinuzzi et al. 2001; Chaudhury and Colwell 2002), and rats (Rudy and Pugh 1998; Valentinuzzi et al. 2004; Winocur and Hasher 1999, 2004). More recently, investigations into the mechanism through which the circadian clock regulates long-term memory formation in Aplysia, have suggested that the circadian clock modulates memory formation in the presynaptic sensory neurons by regulating the induction of kinase activity and learning induced gene transcription (Lyons et al. 2006).

In contrast, it is more difficult to draw conclusions about the impact of the circadian clock on short-term memory (STM). In studies emphasizing short-term memory or working memory tasks in humans, time of day appears to influence memory and performance (reviewed in Schmidt et al. 2007). Unfortunately, these psychological task studies can be difficult to interpret or to identify mechanisms of regulation due to the intertwined impact of sleep-wake cycles, time awake, and the circadian clock (Dijk and von Schantz 2005; Blatter and Cajochen 2007). In animal models of learning and memory, including associative and nonassociative learning in Aplysia (Fernandez et al. 2003; Lyons et al. 2005), contextual fear conditioning (Rudy and Pugh 1998), and social

\footnotetext{
${ }^{3}$ Corresponding author.
}

Email lyons@bio.fsu.edu; fax (850) 644-0989.

Article is online at http://www.learnmem.org/cgi/doi/10.1101/lm.1146009. memory in rats (Reijmers et al. 2001), the circadian clock appears to have no effect on short-term memory. This apparent lack of circadian modulation on STM may be due to the nature of the learning task assayed (e.g., food-related behavior or defensive behavior), or to a masking of the role of the circadian clock caused by the protocol used in training. For most learning and memory studies, strong training protocols are used that induce robust, consistent levels of memory. It is possible that the strength of the training protocols obscured the role of the circadian clock on short-term memory. Indeed, masking of circadian modulation of memory by increasing the strength of the training protocol has been shown for contextual fear conditioning in mice (Chaudhury and Colwell 2002) and for in vitro LTP in hippocampal slices (Chaudhury et al. 2005).

To rigorously analyze the impact of circadian regulation on short-term memory formation, we utilized the Drosophila negatively reinforced olfactory-learning paradigm (Tully and Quinn 1985). In this paradigm, flies learn to associate an odorant (conditioned stimulus +, CS+) with an electric shock (unconditioned stimulus, US), such that the odorant becomes more aversive. The strength of the association and subsequent performance can be modulated by varying the number of pairings between the odorant and electric shock (Beck et al. 2000). A single pairing of the CS+ and the US provides a modest amount of learning and memory formation, whereas 12 shocks paired to a single odorant will provide a performance plateau (Tully and Quinn 1985; Beck et al. 2000).

We found that the circadian clock modulates short-term olfactory memory with peak memory levels in the early to midevening, and the lowest memory levels during midday in both light-dark cycles (LD) and constant darkness (DD). Circadian mutants for the core clock genes, period (per) and timeless (tim), failed to show any rhythms in STM. Circadian gating of sensory perception does not appear to underlie circadian modulation of STM as shock avoidance and olfactory avoidance behaviors were not regulated by time of day. Moreover, we determined that the circadian rhythm in STM appears to be modulated by central brain circadian oscillators and not the peripheral circadian clock in the 
antennae as cryptochrome mutants exhibited strong circadian rhythms in STM. Thus, this study represents the first description of the circadian regulation of short-term memory in a canonical genetic system.

\section{Results}

\section{Circadian rhythms in short-term memory formation}

As a first step in determining if time of day affected STM formation, we investigated short-term olfactory memory during light-dark (LD) cycles using two genotypes of flies, Canton-S and $w^{1118}$. At six time points throughout the LD cycle, flies were trained for negative olfactory conditioning by pairing an aversive odor presentation with electrical shocks (12 shocks, 90 volts with 1 -min odor presentation; Long Program). STM was assessed $3 \mathrm{~min}$ after training. Consistent with previous studies, training and testing were performed using dim red light. Flies 2-5 d old were exclusively used in our experiments to minimize potential differences in the level of memory formation between groups due to age. The concentrations of odors used were individually determined by genotype such that the distribution of naive flies between the odors was balanced (data not shown). We found that both Canton-S and $w^{1118}$ flies demonstrated diurnal rhythms in short-term olfactory learning with peak memory (performance index, PI) observed at ZT 13 and ZT 17 (Fig. 1A,B). Further analysis of the PIs derived separately for benzaldehyde and octanol as the CS+ (half-PIs) revealed that the equivalent diurnal regulation of STM formation occurred for each odor (data not shown), suggesting that responses to an individual odor were not driving the diurnal rhythm.

The difference in STM during the LD cycle could be attributable to either a role for LD cycles in modulating performance in this assay, or due to the regulation of olfactory learning and memory by an endogenous circadian clock. To distinguish between these possibilities, wildtype and $w^{1118}$ flies were trained and tested under constant dark conditions. In Drosophila, the circadian system functions continuously throughout all postembryonic stages, and locomotor activity rhythms of "dark-reared" adult flies can be set through larval entrainment to LD cycles (Sehgal et al. 1992; Kaneko et al. 2000). Consequently, to ensure LD entrainment of very young adults, all fly stocks were cultured continuously in LD cycles during all developmental stages. For both genotypes, flies exhibited a robust circadian rhythm in STM on the first day of DD with peak performance indices observed at CT 13 and CT 17 (Fig. 1C,D).
A

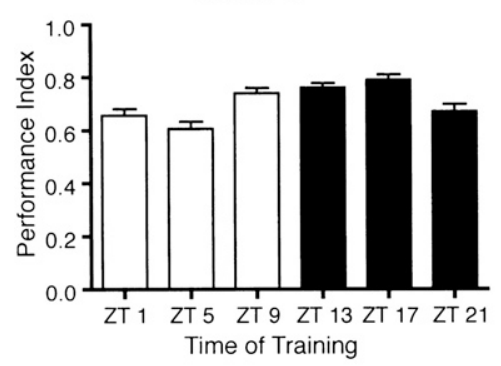

C

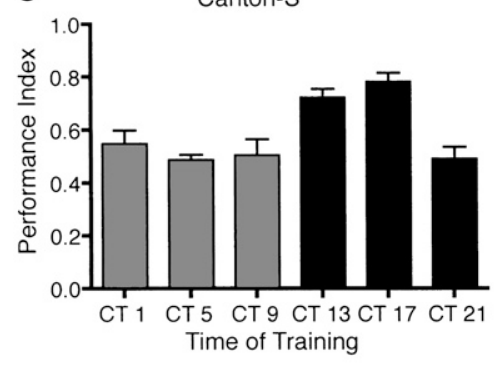

E

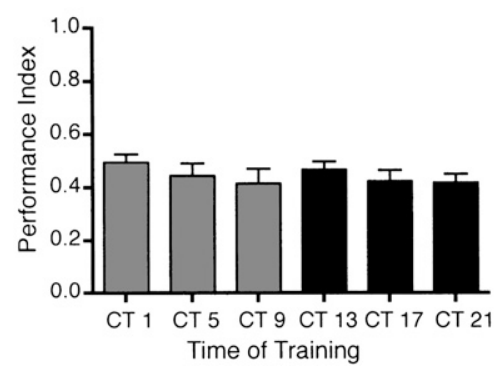

B

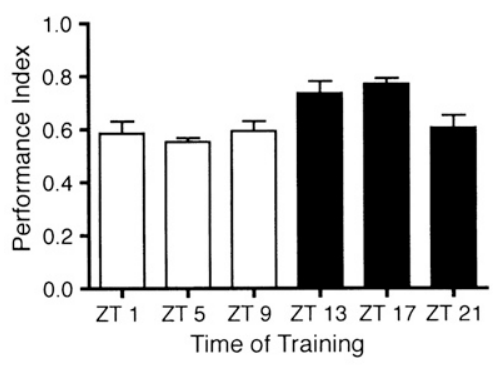

D

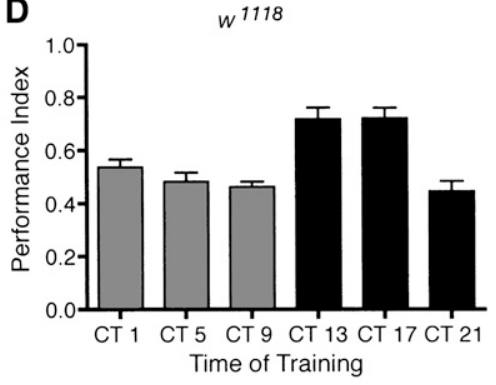

$\mathbf{F}$

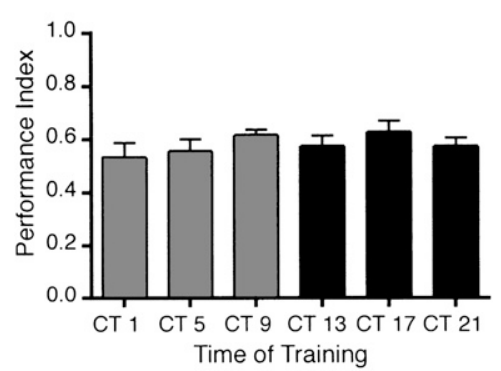

Figure 1. Circadian modulation of short-term olfactory memory. ( $A$ ) Canton-S flies exhibited a significant diurnal rhythm in short-term olfactory memory $(n=8$ groups per time point; one-way ANOVA $F_{(5,42)}=9.01, P<0.001$; Tukey's post-hoc analyses $P<0.05$ for ZT 1 vs. $13, Z \mathrm{ZT} 1$ vs. 17 , ZT 5 vs. 9, ZT 5 vs. 13, ZT 5 vs. 17, ZT 17 vs. 21). Mean performance indices and SEM are plotted. White bars represent times in the light portion of the cycles, while black bars represent training and testing during the dark phase of the LD cycle. $(B) w^{1118}$ flies also exhibited diurnal rhythms in STM with similar peaks in performance observed in the early evening at ZT 13 and ZT 17 ( $n=8$ groups per time point; one-way ANOVA $F_{(5,42)}=5.37, P<0.001$; Tukey's post-hoc analyses $P<0.05$ for ZT 1 vs. 17, ZT 5 vs. 13 , ZT 5 vs. 17, ZT 9 vs. 17, ZT 17 vs. 21). (C,D) Circadian modulation of STM. In DD, the circadian rhythms in STM became more pronounced with Canton-S flies exhibiting significantly greater levels of STM when trained at CT 13 and CT $17\left(C, n=6\right.$ to 8 groups per time point; ANOVA $F_{(5,36)}=10.30, P<0.001$; Tukey's post-hoc analyses $P<0.01$ for CT 1 vs. 17 , CT 5 vs. 13, CT 5 vs. 17, CT 9 vs. CT 13, CT 9 vs. 17 , CT 13 vs. CT 21, CT 17 vs. 21), and for $w^{1118}$ flies significantly greater STM was observed at CT 13 and CT $17\left(D, n=7\right.$ to 8 groups per time point; ANOVA $F_{(5,38)}=12.98, P<0.001$; Tukey's post-hoc analyses $P<0.01$ for CT 13 and CT 17 compared with all other time points). Gray bars represent training during the subjective day, while black bars represent training during the subjective night. $(E, F)$ No rhythm in STM in circadian mutants. per ${ }^{01}$ flies $\left(E, n=6\right.$ to 8 groups per time point; ANOVA $\left.F_{(5,39)}=0.66, P=0.65\right)$ and $\operatorname{tim}^{01}$ flies $\left(F, n=6\right.$ to 8 groups per time point; ANOVA $F_{(5,33)}=0.84, P=0.53$ ) exhibited similar levels of STM when trained at different times throughout the circadian cycle. 
significant, but modest. However, the 12 shock pairings used in the above experiments typically results in plateau levels of performance for 3-min memory (Tully and Quinn 1985; Beck et al. 2000). If the circadian clock was affecting the strength of the association, a higher amplitude rhythm may be found with submaximal levels of training. To test this hypothesis, we analyzed the effect of the circadian clock on olfactory memory using the more sensitive short program training protocol in which a single electric shock (1.25 sec, 90 volts) is paired with short $(10 \mathrm{sec})$ odor presentation (Beck et al. 2000). We found that Canton-S flies trained in LD with a single trial training protocol demonstrated robust STM with peak performance observed at ZT 13 and ZT 17 as previously found with the long program (Fig. 2A). Likewise, Canton-S flies trained on the first day of DD exhibited a highly significant circadian rhythm in olfactory memory with a similarly phased peak (Fig. 2B). These results suggest that in the experiments shown in Figure 1 in which a 12 shock, long odor presentation was used for training, the magnitude of the circadian clock effect on olfactory memory was being partially masked due to the strength of the training protocol. These results also signify that the endogenous clock is most likely modulating the strength of the association formed.

We further investigated the circadian modulation of one trial learning by training flies on the third day of DD. Canton-S flies showed a significant circadian rhythm in STM (Fig. 2C), although the amplitude of the rhythm was dampened compared with flies trained on the first day of DD. Potentially, the observed dampening of the amplitude with extended time in DD may be due to desynchronization or phase misalignment between circadian oscillatory neurons. If the circadian rhythm in olfactory memory is modulated by multiple groups of oscillatory neurons, either separately or jointly modulating aspects of the phase of the rhythm, then desynchronization between oscillators may result in weaker or dampened circadian rhythms as the peaks and troughs become less well-defined. Flies were not kept for more extended periods in DD due to the necessity of age matching flies between learning and memory experiments.

In constant light conditions (LL), the circadian system of Drosophila appears to be disrupted, as evidenced by arrhythmic locomotor activity (Konopka et al. 1989; Hamblen-Coyle et al. 1992; Power et al. 1995; Yoshii et al. 2005) and disrupted or dampened molecular oscillations in core clock gene expression (Price et al. 1995; Marrus et al. 1996; Qiu and Hardin 1996; Yoshii et al. 2005). We next considered whether the rhythm in STM, as an output of the circadian clock, was also abolished in LL. We confirmed that under our LL conditions locomotor activity of wild-type flies was arrhythmic. We entrained Canton-S flies to LD, released them into LL, and assessed individual locomotor activity rhythms. As expected, 26 of 27 flies were arrhythmic when the second to seventh days of LL were analyzed. To assess the effect of LL on STM, wild-type flies were entrained to LD cycles, transferred to LL and then trained for olfactory conditioning using the single shock paradigm on the fourth day of LL. As predicted, no rhythm in STM was observed for Canton-S flies in LL (Fig. 2D). Under LL conditions, the performance indices were intermediate to the peaks and trough levels observed for flies trained in DD.

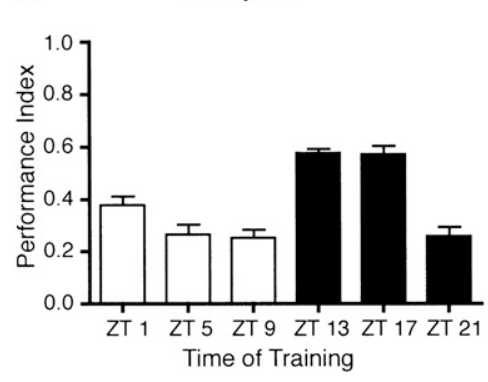

B
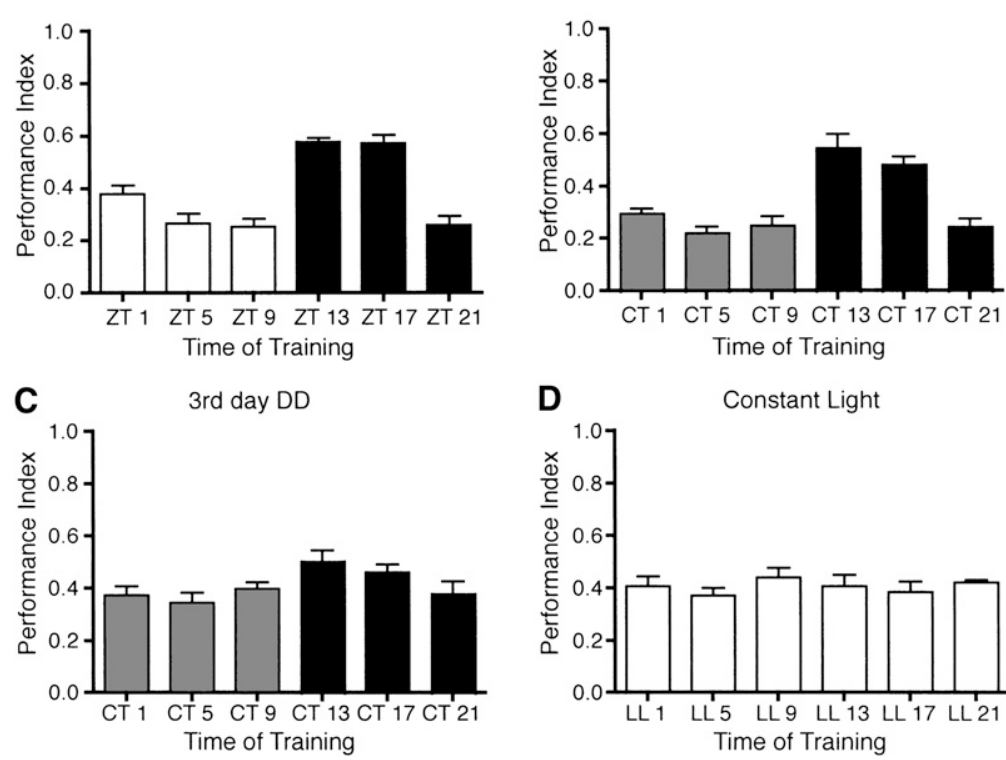

D

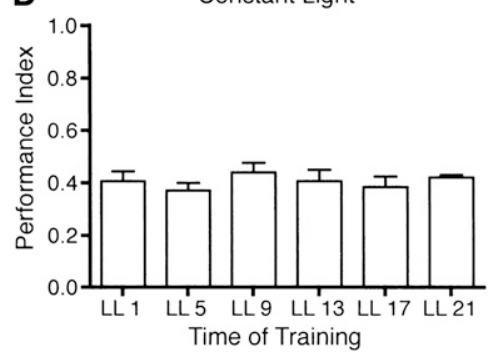

Figure 2. Circadian rhythms in one trial learning. Canton-S flies were entrained to LD cycles and then trained and tested for olfactory conditioning at different times using a single 1.25-sec (90 V) electric shock delivered toward the end of a 10-sec CS+ odor presentation in $(A) \mathrm{LD}$ cycles, $(B, C) \mathrm{DD}$, and $(D) \mathrm{LL}$ conditions. (A) In LD, single-trial olfactory conditioning induced strong diurnal rhythms in STM with flies exhibiting significantly greater memory when trained at ZT 13 and ZT 17 ( $n=8$ groups per time point; ANOVA $F_{(5,43)}=22.62, P<0.001$; Tukey's post-hoc analyses $P<0.01$ for ZT 13 and ZT 17 compared with all other time points). (B) Similarly, in DD, single-trial olfactory conditioning induced robust circadian rhythms in STM with significantly greater memory observed when flies were trained at CT 13 and CT $17\left(n=8\right.$ groups per time point; ANOVA $F_{(5,42)}=19.36, P<0.001$; Tukey's post-hoc analyses $P<0.01$ for $C T 13$ and CT 17 compared with all other time points). (C) Circadian rhythms in STM were free-running with extended time in DD. Canton-S flies exhibited significant circadian rhythms in STM on the third day of DD with significantly decreased memory formation observed when flies were trained at CT 5 ( $n=8$ groups per time point; ANOVA, $F_{(5,43)}=2.79, P<0.05$; Tukey's post-hoc analyses $P<0.05$ for CT 5 vs. CT 13). (D) Constant light abolished the rhythm in STM. Flies trained and tested on the fourth day of LL exhibited no rhythms in STM ( $n=8$ groups per time point; ANOVA $\left.F_{(5,42)}=0.51, P=0.77\right)$.

\section{The circadian oscillator does not modulate STM through sensory gating}

The ability of the animal to form associative memories for classical olfactory learning depends upon the animal's perception of both the odor presentation (conditioned stimulus, CS) and the unconditioned stimulus (US), the electric shock. Potentially, circadian rhythms in either odor or shock perception, i.e., circadian gating of sensory perception, could underlie the observed rhythms in STM. Circadian rhythms in sensory perception have been reported for olfactory sensitivity in Drosophila, cockroach, moths, and mice (Krishnan et al. 1999; Page and Koelling 2003; GranadosFuentes et al. 2004, 2006; Silvegren et al. 2005; Rymer et al. 2007) and gating of light sensitivity in Drosophila larvae (Mazzoni et al. 2005). Given the widespread circadian modulation of sensory stimuli, we tested whether the rhythms in sensory perception at the behavioral level under the conditions used in our experiments were responsible for the rhythms in short-term olfactory memory that we observed. We tested responses to the unconditioned stimulus by assessing shock avoidance for Canton-S and $w^{1118}$ 
flies at six time points during the light-dark cycle. Neither genotype exhibited significant time-of-day differences in their response to the electric shocks (Fig. 3). Thus, it does not appear that underlying rhythms in response to the unconditioned stimulus are responsible for the diurnal rhythm observed for STM.

We next examined whether the circadian rhythm in olfactory memory was due to underlying circadian regulation of olfactory sensitivity. Circadian regulation of olfactory responsiveness in Drosophila has been shown through numerous electrophysiological studies of antennal responses by Hardin and colleagues, and more recently, at the behavioral level (Zhou et al. 2005). Interestingly, circadian regulation of olfactory avoidance behavior appears to vary depending upon both the odor used and the concentration (Zhou et al. 2005). Consequently, we investigated whether the circadian clock regulated olfactory responsiveness at the behavioral level for Canton-S and $w^{1118}$ flies with the parameters, i.e., odor concentrations and times, used to train and test flies for short-term olfactory memory. We found no significant differences with respect to time of day in the behavioral odor avoidance responses for either CS or $w^{1118}$ flies under the conditions used for training for short-term olfactory conditioning (Fig. 4). The lack of a rhythm in odor avoidance behavior at the concentrations used for our STM experiments for benzaldehyde or octanol for either genotype strongly suggests that circadian regulation of olfactory sensitivity is not the underlying determinant behind the rhythms we observed in short-term olfactory learning. Moreover, the rhythms in olfactory sensitivity previously shown in Drosophila using electroantennagram recordings identify a narrow window of peak olfactory sensitivity around ZT/ CT 17 (Krishnan et al. 1999). In contrast, we observed a much broader peak in olfactory memory with performance indices of similar magnitude observed at CT 13 and CT 17. Thus, the above experiments suggest that circadian modulation of olfactory memory occurs downstream from the flies' initial response to the conditioned and unconditioned stimuli. However, the previous experiments do not rule out peripheral antennal oscillators as the site of circadian modulation of olfactory memory.

Independent peripheral oscillators located in the antennae in Drosophila or in the olfactory bulb of mice are both necessary and sufficient for the circadian regulation of olfactory sensitivity or olfactory neuronal firing rate (Tanoue et al. 2004; GranadosFuentes et al. 2006). Although we did not observe any rhythms in our avoidance assays, the peripheral circadian clock in the antennae could regulate the rhythm in STM formation by differentially regulating neuronal firing rate or downstream signaling, etc., such that the strength of the associative memory formed was affected by time of day.

To definitively test whether the antennal circadian oscillator was necessary for the circadian rhythm in olfactory memory, we investigated whether circadian rhythms persisted in cryptochrome mutant flies in which the peripheral antennal circadian oscillators are nonfunctional, but the central pacemakers remain oscillatory. In Drosophila pacemaker neurons (central brain oscillators), CRYPTOCHROME (CRY), a flavin/pterin containing protein, acts as a blue-light photoreceptor and a component of the light entrainment pathway. $c r y^{b}$ flies entrain to LD cycles and exhibit rhythmic locomotor activity under LD, DD, and LL conditions as well as maintaining molecular oscillations of core clock components in central brain oscillatory cells (Emery et al. 1998; Stanewsky et al. 1998; Ivanchenko et al. 2001; Collins et al. 2006). The situation in the central brain pacemaker neurons stands in stark contrast to the situation in peripheral oscillators in the antennae, Malphigian tubules, and the eye. In peripheral oscillators, dCRY acts as a circadian transcriptional repressor and a necessary component of the circadian oscillator (Ivanchenko et al. 2001; Krishnan et al. 2001; Hardin 2005; Collins et al. 2006), similar to its role in the mammalian circadian clock (Kume et al. 1999; van der Horst et al. 1999). In antennal oscillators, $c r y^{b}$ mutants fail to show any rhythms in olfactory sensitivity (Krishnan et al. 2001; Hardin 2005). Taking advantage of the differential role of CRY between central and peripheral oscillators, we used $c r y^{b}$ mutant flies to test whether the antennal circadian oscillators were necessary for circadian modulation of STM. We entrained $c r y^{b}$ mutants (in wild-type Canton-S background and $w^{1118} c r y^{b}$ ) to LD cycles, switched them to DD, and then trained them using the long protocol for olfactory conditioning on the first day of DD during the subjective day and during the subjective night. Surprisingly, the $c r y^{b}$ mutants exhibited circadian rhythms in STM with similar amplitude and peak performance to control flies (Fig. 5). Thus, the antennal circadian oscillator is not necessary for circadian modulation of olfactory memory. This strongly suggests that central brain pacemakers regulate the rhythms in short-term olfactory memory.

\section{Discussion}

Learning represents a change in an animal's behavior in response to particular events or stimuli. The extent of learning and the degree of memory formed can be modulated by factors both internal and external to the animal. Consequently, in order to understand the mechanisms through which memory formation occurs, it is necessary to characterize the factors which can modulate learning and memory formation. The circadian clock broadly impacts long-term learning and memory through several different mechanisms. First, classical time-stamping has been demonstrated in many species, and in many types of learning including conditioned taste aversion in crabs (Pereyra et al. 1996), food foraging behavior in bees, and for learning in hamsters (Ralph et al. 2002; Cain et al. 2004). In these cases, circadian time represents a feature of learning rather than a modulator of learning and memory. Second, disruption or phase shifting of the circadian clock adversely impacts memory. For example, circadian disruption results in decreased cognition and performance such as seen in chronic jet lag (Cho et al. 2000; 
A

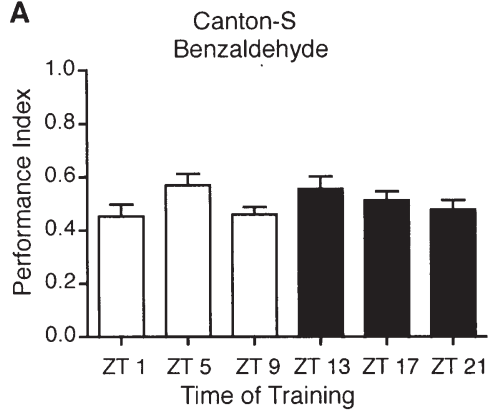

C

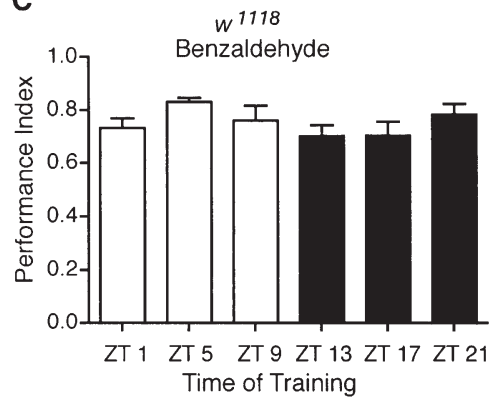

B

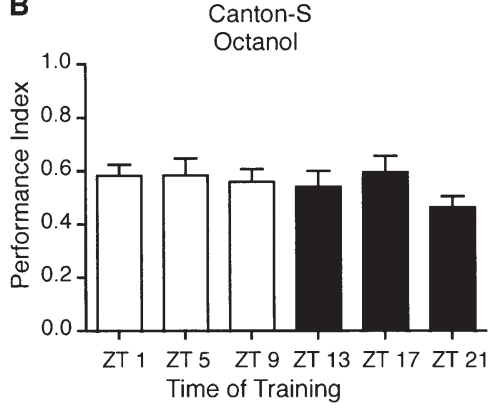

D

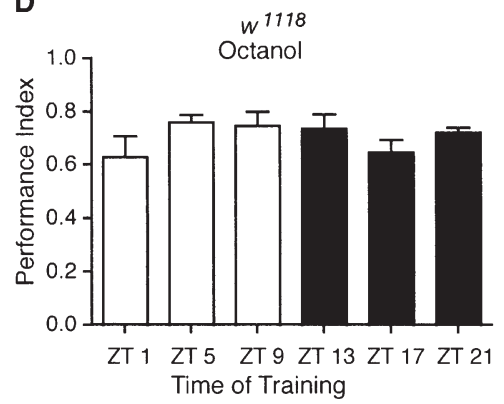

Figure 4. No rhythms in odor avoidance behavior were found. Flies entrained to LD cycles were tested for odor avoidance responses in the T-maze at the indicated times. A performance index was calculated for the avoidance of the indicated odorants. $(A, B)$ No significant time-of-day differences were observed in odor avoidance behavior for Canton-S for either $(A)$ benzaldehyde $(n=8$ to 14 groups per time point; ANOVA $\left.F_{(5,57)}=1.42, P=0.23\right)$ or $(B)$ octanol $(n=8$ to 14 groups per time point; ANOVA $\left.F_{(5,59)}=1.08, P=0.38\right)$. $(C, D)$ No significant time-of-day differences were observed in odor avoidance behavior for $w^{1118}$ flies for either $(C)$ benzaldehyde $\left(n=8\right.$ to 10 groups per time point; ANOVA $F_{(5,51)}=$ $1.44, P=0.23)$ or $(D)$ octanol $\left(n=8\right.$ to 10 groups per time point; ANOVA $\left.F_{(5,48)}=1.14, P=0.35\right)$.

Cho 2001) or circadian disruption can induce retrograde amnesia (Tapp and Holloway 1981; Fekete et al. 1985). Finally, the circadian clock modulates learning such that greater memory is formed or recalled at a particular circadian phase as has been well characterized in Aplysia and zebrafish (Fernandez et al. 2003; Lyons et al. 2005, 2006; Rawashdeh et al. 2007). In contrast, how time of day affects short-term memory is considerably less well understood and often dismissed as having little or no impact.

Nevertheless, circadian modulation of short-term memory represents an important area of research since short-term behavioral changes comprise a component of many operant-learning paradigms, and in humans, cognitive performance frequently relies upon short-term learning and memory. The present research significantly extends our understanding of memory modulation and provides the groundwork for future research by demonstrating circadian regulation of short-term memory using a classical learning paradigm in a genetically amenable model system. We have shown that the circadian clock modulates short-term olfactory memory in Drosophila with higher levels of memory observed in the early to midportion of the night. Moreover, the rhythm in olfactory memory is dependent upon circadian modulation of learning, rather than modulation of the flies' responsiveness to sensory stimuli. Importantly, we found that the antennal circadian oscillator does not affect the circadian rhythm in STM, suggesting that brain circadian oscillators modulate memory formation as an output.

Where is the central circadian clock that modulates memory formation? Investigations by many laboratories, using diverse techniques, have demonstrated that the mushroom bodies (MBs) represent a critical site necessary for olfactory learning (de Belle and Heisenberg 1994; Connolly et al. 1996; Zars et al. 2000; responsiveness to stimuli in sensory neurons, (2) modulation of stimuli strength or perception in neurons responsible for associative memory formation, and (3) modulation of memory formation. Recall or retrieval of the memory, although more often considered a site of modulation with respect to intermediate or long-term memory, could also be affected by the circadian clock.

We investigated whether circadian rhythms in sensory sensitivity were responsible for the circadian rhythm we observed in olfactory STM. Circadian rhythms in olfactory sensitivity have been well characterized in Drosophila (Krishnan et al. 1999, 2001; Tanoue et al. 2004) and in cockroaches (Page and Koelling 2003). Furthermore, the circadian gating of sensory input or sensory sensitivity appears to encompass multiple sensory systems, as circadian pacemaker neurons in Drosophila larvae gate the sensitivity of larvae to light (Mazzoni et al. 2005). In avoidance behavior assays, we found that neither avoidance behavior in response to electric shock nor to odor presentation was regulated by the circadian clock. It should be noted that in contrast to our results, Zhou et al. (2005) reported a low amplitude circadian rhythm in olfactory avoidance behavior to repulsive and attractive concentrations of 4-methylcyclohexanol in a similar assay. However, considerable differences exist between the two studies regarding the odors tested and the concentrations used. Most likely, under the conditions used in our experiments, the concentration of odors used for training was such that the odors were highly salient to the animals at all times of day so that no time-of-day effects were observed for odor avoidance. Additionally, in our experiments significant levels of STM were induced using both a short and long training odor presentation, suggesting that olfactory sensitivity or odor perception was not a factor. Therefore, we conclude that circadian modulation of primary sensory 
A

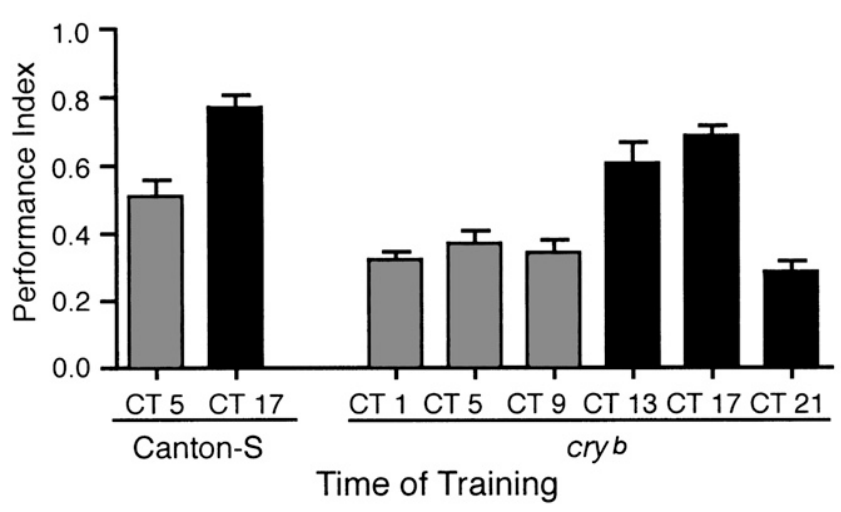

B

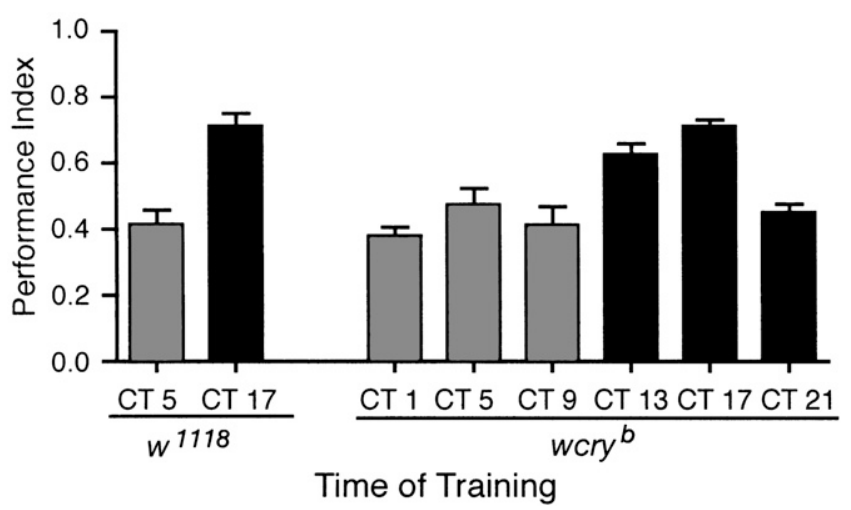

Figure 5. Cryptochrome mutants maintain circadian rhythms in olfactory memory. Flies were entrained to LD cycles, transferred to DD, and then trained for olfactory conditioning using the 12 shock long protocol. $c r y^{b}$ mutants exhibited circadian rhythms in STM with similar amplitude and peak performance to control flies. $(A)$ Canton-S and $c r y^{b}$ flies $(n=6$ to 8 groups per time point; ANOVA $F_{(7,42)}=19.91, P<0.001$; Tukey's posthoc analyses $P<0.01$ for CS CT 5 vs. CT 17 and $P<0.01$ for $c r y$ mutant flies CT 13 and CT 17 compared with all time points at which $c r y^{b}$ flies were tested). (B) $w^{1118}$ and $w c r y^{b}$ flies $(n=6$ to 8 groups per time point; ANOVA $F_{(7,45)}=12.20, P<0.001$; Tukey's post-hoc analyses $P<0.01$ for $w^{1118}$ CT 5 vs. Ct 17 and $P<0.05$ for $w_{c r y}^{b}$ flies at CT 13 and CT 17 compared with all other time points at which $w_{c r y}{ }^{b}$ flies were tested).

responses is not the principal mechanism through which the circadian clock modulates STM.

Nonassociative effects of stimuli presentation can affect the performance of flies within the olfactory-learning paradigm (Acevedo et al. 2007a,b). Habituation or sensitization to a noxious stimulus could be regulated by the circadian clock so that while the same training protocol may be used at all times of day, the salience of the US or the aversive odor used as the CS+ may be different. Recent research has shown habituation to electric shock occurs in flies and is dependent upon and modulated by the MBs (Acevedo et al. 2007a). Similarly, electric shock can induce nonassociative decreases in olfactory responsiveness (Acevedo et al. 2007b). Furthermore, habituation of an olfactory startle response also occurs in Drosophila, although it remains unknown in what part of the olfactory circuit habituation occurs (Cho et al. 2004; Wolf et al. 2007). If the circadian clock modulates habituation, to either electric shock or odor presentation, one would predict that a long training protocol would result in circadian rhythms of STM while a short training protocol, for which habituation should not occur, would not show a rhythm in STM. However, in our experi- ments, we found that circadian modulation of olfactory memory appeared much stronger with a higher amplitude circadian rhythm after a single trial learning protocol. These results suggest that the circadian clock is not modulating STM through habituation to the US or to odor presentation, but rather that the effect of the circadian clock was being partially masked in the experiments using a stronger training protocol.

We adduce that the circadian clock is modulating associative learning and memory, at least in part, by modulating the strength of the association during memory formation. Due to our experimental design and behavioral analysis of short-term memory, we cannot exclude the possibility that the circadian clock also affects memory recall. The availability of second messenger or downstream signaling cascades also may be regulated by the circadian clock as manipulation of the cAMP cascade has been shown to cause a reduction in STM (Nighorn et al. 1991; Han et al. 1992). Although it is possible that the circadian clock modulates the stability of the memory, we find this scenario unlikely, since we were assessing three-minute memory in these experiments. In addition, in all experiments, the training protocols used induced significant STM with time of day modulating the magnitude of STM. As the study of circadian modulation of memory progresses in the future, it will be extremely interesting to determine whether the circadian clock affects middle- or long-term memory via modulation of memory stability or recall.

What is the function of circadian modulation of memory? Circadian modulation of long-term memory formation appears linked to the phase of the animal's activity period for both invertebrates and vertebrates. Given the increased exposure to sensory stimuli during active periods as well as behavioral activity such as food foraging or predator avoidance, there are likely advantages in coordinating learning with the phase of the animal's activity. For example, the diurnal Aplysia californica exhibits greater memory formation for associative learning during the day, while the nocturnal Aplysia fasciata is more capable at night (Lyons et al. 2005). Likewise, early morning active snails exhibit higher scores in conditioned taste aversion earlier in the day compared with the afternoon (Wagatsuma et al. 2004). Examples of higher levels of long-term learning correlated with activity period are also seen in vertebrate animals including zebrafish, mice, and rats (Valentinuzzi et al. 2001, 2004; Rawashdeh et al. 2007). Thus, the emerging picture of circadian modulation of long-term memory formation indicates that this is a widespread phenomenon conserved across phylogeny as well as types of learning. On the other hand, circadian modulation of short-term memory formation appears to be a less widespread phenomenon highly dependent upon the type of learning behavior assayed.

Initially, one might be surprised by the phase of the circadian rhythm in olfactory memory with peak levels of STM seemingly occurring during the flies' resting or sleeping periods. Sleep in Drosophila exhibits many of the characteristics as sleep in higher organisms including circadian cycling, homeostatic regulation, sleep rebound, preferred posture for rest, and a state of decreased responsiveness to external stimuli (Hendricks et al. 2000; Shaw et al. 2000; Huber et al. 2004). Indeed, consideration of the role of sleep in learning and memory may be further warranted by the dual regulatory function of $\mathrm{MB}$ neurons in both sleep and olfactory memory. Recent studies have demonstrated that ablation of the MBs or changes in neuronal activity within the MB neurons result in reduced sleep or altered sleep characteristics (Joiner et al. 2006; Pitman et al. 2006). However, Drosophila sleep occurs in a fragmented, bout pattern (Hendricks et al. 2000; Shaw et al. 2000; Huber et al. 2004). Consequently, it would be erroneous to state that the peak levels of STM we observed occur while the flies are sleeping, as sleep bouts occur throughout the 
day and the night albeit with a greater amount of sleep occurring at night. Thus, while it will be of considerable interest in future studies to compare MB regulation of sleep and MB regulation of olfactory memory with circadian activity, we do not think that interruptions in sleep underlie the circadian rhythms we observed in short-term olfactory memory.

Although the broad peak in Drosophila short-term olfactory memory does not appear to coincide with the more narrow peak in locomotor activity as observed in the laboratory, there may indeed be a link between circadian regulation of activity and olfactory memory. Drosophila males in isolation display biphasic crepuscular behavioral rhythms with activity peaks in the late day/early evening and then a second peak of activity in anticipation of dawn. However, Drosophila heterosexual couples display much different activity rhythms with low activity near dusk and then high levels of activity throughout the night with increased courtship (Fujii et al. 2007). Social context also has previously been shown to influence circadian resetting (Levine et al. 2002), and group composition may affect the phase of circadian locomotor activity or rest-activity cycles. During our olfactory learning assay, the flies were housed, trained, and tested in groups of $\sim 50$. Hence, these animals would not be expected to behave as flies in isolation, but rather as flies in a social context. With little information available regarding activity rhythms of natural populations, groups of flies may exhibit broad peaks of activity including both increased locomotor activity around dusk and increased activity and courtship throughout much of the night, similar to that found in heterosexual couples (Fujii et al. 2007). Presumably, there are advantages in increased olfactory memory with regard to both higher levels of locomotor activity and courtship behavior. Selective pressures related to these increases in nighttime locomotor activity and courtship may have shaped the circadian rhythm with the broad nighttime peak observed in short-term olfactory memory.

On the other hand, it is possible that the circadian rhythm of olfactory memory is not regulated through the enhancement of memory performance at night, but rather through the regulation of the trough phase of the rhythm. From this perspective, olfactory memory may be suppressed during the day when locomotor activity levels are fairly low, with decreases in memory formation evolving when and to the degree that the organisms could tolerate. In our experiments in which flies were trained and tested after several days in DD (Fig. 2C), the dampening in the amplitude of the rhythm appeared to be due to an increase in the trough levels of olfactory memory. If coordination between multiple oscillatory neurons regulated suppression of short-term memory formation, then it is likely that desynchronization between oscillatory neurons may lead to increased memory formation during the trough phase of the rhythm. Thus, potentially the same selective pressures that shaped the trough phases in the circadian rhythms of locomotor activity and olfactory sensitivity have also effected the trough phases of circadian modulation of olfactory memory.

Insight into the function of the circadian rhythm in olfactory memory may be gained through analysis of how the circadian clock regulates a suite of coordinated or interlinked behaviors and rhythms. In either of the above scenarios, the presumption is that the circadian clock is modulating the peak or the trough phase (or both) of the rhythm in olfactory memory in coordination with circadian regulation of locomotor and social activity. In future studies, it will be interesting to analyze interacting behaviors to determine how alterations in the core circadian oscillator, e.g., in short-period or long-period mutants, affect the phase relationship between different behaviors and circadian rhythms.

Alternatively, the rhythm in short-term olfactory memory may be associated with rhythmic environmental events or a pred- ators' circadian cycle as has been suggested for the rhythm in olfactory sensitivity (Krishnan et al. 1999). Recently, researchers at Vanderbilt University demonstrated that olfactory memory in the nocturnal cockroach Leucophaea maderae is modulated by the circadian clock with a similarly phased peak in performance (Decker et al. 2007) as that shown here for Drosophila. The phase similarity of peak performance between the studies is intriguing given the differences in the activity profiles between the two model systems, and gives credence to the possibility that the phase of the rhythm in olfactory memory is not dependent solely upon the animal's locomotor activity cycle. Future investigations in this area of research will undoubtedly further our understanding of how the circadian clock regulates output behaviors and may also uncover previously unknown environmental or ecological pressures that could be mitigated by increased short-term memory formation in the early-mid portions of the night.

Circadian modulation of cognitive performance, in addition to interrelated imbalances in sleep-wake homeostasis, can have tremendous impact in many occupations including shift-work, the medical profession, and the transportation industry. This study represents the first demonstration of circadian modulation of short-term memory in a genetically tractable model system, and sets the stage for future studies investigating the molecular and cellular mechanisms through which the circadian clock modulates short-term memory.

\section{Materials and Methods}

Flies were raised on cornmeal, sucrose, and yeast agar at $25^{\circ} \mathrm{C}$ in $12: 12 \mathrm{~h}$ light/dark cycles. Zeitgeber Time (ZT) 0 represents dawn (lights on), while ZT 12 refers to dusk (lights off).

\section{Classical olfactory conditioning}

Classical olfactory conditioning and avoidance behavior testing was performed under dim red light at $25^{\circ} \mathrm{C}$ as previously described using a T-maze apparatus similar to other studies (Tully and Quinn 1985; Ferris et al. 2006). Briefly, young flies (2-5 d old) were trained in groups at different times of the light-dark cycle or under constant conditions. Experiments during the dark portion of the cycle or under constant conditions were performed under dim red light with flies never exposed to bright or white light following the entrainment period. In most experiments, flies were trained using a long program in which the unconditioned stimulus consisted of 12 electric shocks ( $1.25 \mathrm{sec}, 90$ volts, $1 \mathrm{~min})$ delivered through a copper grid simultaneously with exposure to the CS+ odor. After odor/shock presentation, flies were exposed to fresh air for $30 \mathrm{sec}$, followed by a 1-min exposure to the CS- odor. Flies were then exposed to fresh air for $30 \mathrm{sec}$ and then transferred to the main part of the T-maze for one minute. For STM testing, flies were exposed to a choice between odorants in the T-maze for $2 \mathrm{~min}$.

3-Octanol (Fluka brand from Sigma-Aldrich) and Benzaldehyde (Sigma-Aldrich) were diluted in $10 \mathrm{~mL}$ of light mineral oil (Sigma) and then air was bubbled through the odorant into the Tmaze. Airflow was maintained at $500 \mathrm{~mL} / \mathrm{min}$ for all procedures. Odor concentrations for each genotype were determined by odor balancing such that naïve flies when presented with a choice evenly distributed between odorants.

The performance index was calculated by counting the number of flies choosing the CS- tube and subtracting the number of flies in CS+ tube and then dividing that number by the total number of flies in both. All experiments were run in duplicate mazes with the odor used for the CS+ alternated between mazes and between experiments. The one-half PI was calculated for each maze and then averaged to determine the PI for the experiment. One-way analysis of variance (ANOVA) was used to determine time-of-day effects for all behavioral assays with Tukey post-hoc analysis. $P$ values $<0.05$ were considered statistically significant. 
In olfactory avoidance assays, flies were exposed to individually presented odors through one arm of the T-maze apparatus with fresh air bubbled through mineral oil flowing through the other arm of the T-maze at different times in the light-dark cycle. The odor avoidance response of the flies was calculated similarly to the performance index described above.

\section{Analysis of locomotor activity rhythms}

Locomotor activity of adult male Canton-S flies was monitored as previously described (Tanoue et al. 2004; Benito et al. 2007). Flies were entrained in LD cycles at $25^{\circ} \mathrm{C}$ for $3 \mathrm{~d}$ and then transferred into either LL or DD. Locomotor activity was monitored continuously during entrainment and then for the second through seventh day in constant conditions using Drosophila activity monitors (Trikinetics). Activity data was analyzed by periodogram analysis using the Fly Activity Analysis Suite (FaasX, version 0.7.1; M. Boudinot, Center National de la Recherché Scientifique-Institut Neurobiologie Alfred Fessard). Flies showing a minimum power of 10 and width of 2 in periodogram analysis were considered rhythmic.

\section{Acknowledgments}

The authors thank Dr. Julianna Benito for technical assistance with the Drosophila locomotor activity assays and Dr. Paul Hardin (Texas A\&M University) for generously providing fly stocks.

\section{References}

Acevedo, S.F., Froudarakis, E.I., Kanellopoulos, A., and Skoulakis, E.M. 2007a. Protection from premature habituation requires functional mushroom bodies in Drosophila. Learn. Mem. 14: 376-384.

Acevedo, S.F., Froudarakis, E.I., Tsiorva, A.A., and Skoulakis, E.M. 2007b. Distinct neuronal circuits mediate experience-dependent, nonassociative osmotactic responses in Drosophila. Mol. Cell. Neurosci. 34: 378-389.

Beck, C.D., Schroeder, B., and Davis, R.L. 2000. Learning performance of normal and mutant Drosophila after repeated conditioning trials with discrete stimuli. J. Neurosci. 20: 2944-2953.

Bell-Pedersen, D., Cassone, V.M., Earnest, D.J., Golden, S.S., Hardin, P.E., Thomas, T.L., and Zoran, M.J. 2005. Circadian rhythms from multiple oscillators: Lessons from diverse organisms. Nat. Rev. Genet. 6: 544-556.

Benito, J., Zheng, H., and Hardin, P.E. 2007. PDP1ع functions downstream of the circadian oscillator to mediate behavioral rhythms. J. Neurosci. 27: 2539-2547.

Blatter, K. and Cajochen, C. 2007. Circadian rhythms in cognitive performance: Methodological constraints, protocols, theoretical underpinnings. Physiol. Behav. 90: 196-208.

Cain, S.W., Chou, T., and Ralph, M.R. 2004. Circadian modulation of performance on an aversion-based place learning task in hamsters Behav. Brain Res. 150: 201-205.

Chaudhury, D. and Colwell, C.S. 2002. Circadian modulation of learning and memory in fear-conditioned mice. Behav. Brain Res. 133: 95-108.

Chaudhury, D., Wang, L.M., and Colwell, C.S. 2005. Circadian regulation of hippocampal long-term potentiation. J. Biol. Rhythms 20: 225-236.

Cho, K. 2001. Chronic "jet lag" produces temporal lobe atrophy and spatial cognitive deficits. Nat. Neurosci. 4: 567-568.

Cho, K., Ennaceur, A., Cole, J.C., and Suh, C.K. 2000. Chronic jet lag produces cognitive deficits. J. Neurosci. 20: $1-5$.

Cho, W., Heberlein, U., and Wolf, F.W. 2004. Habituation of an odorantinduced startle response in Drosophila. Genes Brain Behav. 3: 127-137.

Collins, B., Mazzoni, E.O., Stanewsky, R., and Blau, J. 2006. Drosophila CRYPTOCHROME is a circadian transcriptional repressor. Curr. Biol. 16: 441-449.

Connolly, J.B., Roberts, I.J., Armstrong, J.D., Kaiser, K., Forte, M., Tully, T., and O'Kane, C.J. 1996. Associative learning disrupted by impaired Gs signaling in Drosophila mushroom bodies. Science 274: 2104-2107.

de Belle, J.S. and Heisenberg, M. 1994. Associative odor learning in Drosophila abolished by chemical ablation of mushroom bodies. Science 263: 692-695.

Decker, S., McConnaughey, S., and Page, T.L. 2007. Circadian regulation of insect olfactory learning. Proc. Natl. Acad. Sci. 104: 15905-15910.

Dijk, D.J. and von Schantz, M. 2005. Timing and consolidation of human sleep, wakefulness, and performance by a symphony of oscillators. $J$. Biol. Rhythms 20: 279-290.

Dubnau, J., Grady, L., Kitamoto, T., and Tully, T. 2001. Disruption of neurotransmission in Drosophila mushroom body blocks retrieval but not acquisition of memory. Nature 411: 476-480.
Emery, P., So, W.V., Kaneko, M., Hall, J.C., and Rosbash, M. 1998. CRY, a Drosophila clock and light-regulated cryptochrome, is a major contributor to circadian rhythm resetting and photosensitivity. Cell 95: 669-679.

Fekete, M., van Ree, J.M., Niesink, R.J., and de Wied, D. 1985. Disrupting circadian rhythms in rats induces retrograde amnesia. Physiol. Behav. 34: $883-887$.

Fernandez, R.I., Lyons, L.C., Levenson, J., Khabour, O., and Eskin, A. 2003 Circadian modulation of long-term sensitization in Aplysia. Proc. Natl. Acad. Sci. 100: 14415-14420.

Ferris, J., Ge, H., Liu, L., and Roman, G. 2006. G(o) signaling is required for Drosophila associative learning. Nat. Neurosci. 9: 1036-1040.

Fujii, S., Krishnan, P., Hardin, P., and Amrein, H. 2007. Nocturnal male sex drive in Drosophila. Curr. Biol. 17: 244-251.

Granados-Fuentes, D., Saxena, M.T., Prolo, L.M., Aton, S.J., and Herzog, E.D. 2004. Olfactory bulb neurons express functional, entrainable circadian rhythms. Eur. J. Neurosci. 19: 898-906.

Granados-Fuentes, D., Tseng, A., and Herzog, E.D. 2006. A circadian clock in the olfactory bulb controls olfactory responsivity. J. Neurosci. 26: 12219-12225

Hamblen-Coyle, M.J., Wheeler, D.A., Rutila, J.E., Rosbash, M., and Hall, J.C. 1992. Behavior of period-altered circadian rhythms mutants of Drosophila in light:dark cyles (Diptera: Drosophilidae). J. Insect Behav. 5: 417-446.

Han, P.L., Levin, L.R., Reed, R.R., and Davis, R.L. 1992. Preferential expression of the Drosophila rutabaga gene in mushroom bodies, neural centers for learning in insects. Neuron 9: 619-627.

Hardin, P.E. 2005. The circadian timekeeping system of Drosophila. Curr. Biol. 15: R714-R722.

Hendricks, J.C., Finn, S.M., Panckeri, K.A., Chavkin, J., Williams, J.A., Sehgal, A., and Pack, A.I. 2000. Rest in Drosophila is a sleep-like state. Neuron 25: 129-138.

Houl, J.H., Yu, W., Dudek, S.M., and Hardin, P.E. 2006. Drosophila CLOCK is constitutively expressed in circadian oscillator and non-oscillator cells. J. Biol. Rhythms 21: 93-103.

Huber, R., Hill, S.L., Holladay, C., Biesiadecki, M., Tononi, G., and Cirelli, C. 2004. Sleep homeostasis in Drosophila melanogaster. Sleep 27: 628-639.

Ivanchenko, M., Stanewsky, R., and Giebultowicz, J.M. 2001. Circadian photoreception in Drosophila: Functions of cryptochrome in peripheral and central clocks. J. Biol. Rhythms 16: 205-215.

Joiner, W.J., Crocker, A., White, B.H., and Sehgal, A. 2006. Sleep in Drosophila is regulated by adult mushroom bodies. Nature 441: 757-760.

Kaneko, M. and Hall, J.C. 2000. Neuroanatomy of cells expressing clock genes in Drosophila: Transgenic manipulation of the period and timeless genes to mark the perikarya of circadian pacemaker neurons and their projections. J. Comp. Neurol. 422: 66-94.

Kaneko, M., Hamblen, M.J., and Hall, J.C. 2000. Involvement of the period gene in developmental time-memory: Effect of the perShort mutation on phase shifts induced by light pulses delivered to Drosophila larvae. J. Biol. Rhythms 15: 13-30.

Konopka, R.J., Pittendrigh, C., and Orr, D. 1989. Reciprocal behaviour associated with altered homeostasis and photosensitivity of Drosophila clock mutants. J. Neurogenet. 6: 1-10.

Krashes, M.J., Keene, A.C., Leung, B., Armstrong, J.D., and Waddell, S. 2007 Sequential use of mushroom body neuron subsets during Drosophila odor memory processing. Neuron 53: 103-115.

Krishnan, B., Dryer, S.E., and Hardin, P.E. 1999. Circadian rhythms in olfactory responses of Drosophila melanogaster. Nature 400: 375-378.

Krishnan, B., Levine, J.D., Lynch, M.K., Dowse, H.B., Funes, P., Hall, J.C., Hardin, P.E., and Dryer, S.E. 2001. A new role for cryptochrome in a Drosophila circadian oscillator. Nature 411: 313-317.

Kume, K., Zylka, M.J., Sriram, S., Shearman, L.P., Weaver, D.R., Jin, X., Maywood, E.S., Hastings, M.H., and Reppert, S.M. 1999. mCRY1 and mCRY2 are essential components of the negative limb of the circadian clock feedback loop. Cell 98: 193-205.

Levine, J.D., Funes, P., Dowse, H.B., and Hall, J.C. 2002. Resetting the circadian clock by social experience in Drosophila melanogaster. Science 298: 2010-2012.

Lyons, L.C., Rawashdeh, O., Katzoff, A., Susswein, A.J., and Eskin, A. 2005. Circadian modulation of complex learning in diurnal and nocturnal Aplysia. Proc. Natl. Acad. Sci. 102: 12589-12594.

Lyons, L.C., Collado, M.S., Khabour, O., Green, C.L., and Eskin, A. 2006. The circadian clock modulates core steps in long-term memory formation in Aplysia. J. Neurosci. 26: 8662-8671.

Marrus, S.B., Zeng, H., and Rosbash, M. 1996. Effect of constant light and circadian entrainment of perS flies: Evidence for light-mediated delay of the negative feedback loop in Drosophila. EMBO J. 15: 6877-6886

Mazzoni, E.O., Desplan, C., and Blau, J. 2005. Circadian pacemaker neurons transmit and modulate visual information to control a rapid behavioral response. Neuron 45: 293-300. 
McGuire, S.E., Le, P.T., and Davis, R.L. 2001. The role of Drosophila mushroom body signaling in olfactory memory. Science 293: 1330-1333.

Nighorn, A., Healy, M.J., and Davis, R.L. 1991. The cyclic AMP phosphodiesterase encoded by the Drosophila dunce gene is concentrated in the mushroom body neuropil. Neuron 6: 455-467.

Page, T.L. and Koelling, E. 2003. Circadian rhythm in olfactory response in the antennae controlled by the optic lobe in the cockroach. J. Insect Physiol. 49: 697-707.

Pereyra, P., De La Iglesia, H.O., and Maldonado, H. 1996. Training-to-testing intervals different from $24 \mathrm{~h}$ impair habituation in the crab Chasmagnathus. Physiol. Behav. 59: 19-25.

Pitman, J.L., McGill, J.J., Keegan, K.P., and Allada, R. 2006. A dynamic role for the mushroom bodies in promoting sleep in Drosophila. Nature 441: 753-756.

Power, J.M., Ringo, J.M., and Dowse, H.B. 1995. The effects of period mutations and light on the activity rhythms of Drosophila melanogaster. J. Biol. Rhythms 10: 267-280.

Price, J.L., Dembinska, M.E., Young, M.W., and Rosbash, M. 1995. Suppression of PERIOD protein abundance and circadian cycling by the Drosophila clock mutation timeless. EMBO J. 14: 4044-4049.

Qiu, J. and Hardin, P.E. 1996. per mRNA cycling is locked to lights-off under photoperiodic conditions that support circadian feedback loop function. Mol. Cell. Biol. 16: 4182-4188.

Ralph, M.R., Ko, C.H., Antoniadis, E.A., Seco, P., Irani, F., Presta, C., and McDonald, R.J. 2002. The significance of circadian phase for performance on a reward-based learning task in hamsters. Behav. Brain Res. 136: 179. doi: 10.1016/SO166-4328(02)00131-6.

Rawashdeh, O., de Borsetti, N.H., Roman, G., and Cahill, G.M. 2007. Melatonin suppresses nighttime memory formation in zebrafish. Science 318: $1144-1146$.

Reijmers, L.G., Leus, I.E., Burbach, J.P., Spruijt, B.M., and van Ree, J.M. 2001. Social memory in the rat: Circadian variation and effect of circadian rhythm disruption. Physiol. Behav. 72: 305-309.

Rudy, J.W. and Pugh, C.R. 1998. Time of conditioning selectively influences contextual fear conditioning: Further support for a multiple-memory systems view of fear conditioning. J. Exp. Psychol. Anim. Behav. Process. 24: 316-324.

Rymer, J., Bauernfeind, A.L., Brown, S., and Page, T.L. 2007. Circadian rhythms in the mating behavior of the cockroach, Leucophaea maderae. J. Biol. Rhythms 22: 43-57.

Schmidt, C., Collette, F., Cajochen, C., and Peigneux, P. 2007. A time to think: Circadian rhythms in human cognition. Cogn. Neuropsychol. 24: $755-789$

Sehgal, A., Price, J., and Young, M.W. 1992. Ontogeny of a biological clock in Drosophila melanogaster. Proc. Natl. Acad. Sci. 89: 1423-1427.

Shaw, P.J., Cirelli, C., Greenspan, R.J., and Tononi, G. 2000. Correlates of sleep and waking in Drosophila melanogaster. Science 287: 1834-1837.
Silvegren, G., Lofstedt, C., and Qi Rosen, W. 2005. Circadian mating activity and effect of pheromone pre-exposure on pheromone response rhythms in the moth Spodoptera littoralis. J. Insect Physiol. 51: 277-286.

Stanewsky, R., Kaneko, M., Emery, P., Beretta, B., Wager-Smith, K., Kay, S.A. Rosbash, M., and Hall, J.C. 1998. The $c r y^{b}$ mutation identifies cryptochrome as a circadian photoreceptor in Drosophila. Cell 95: 681-692.

Tanoue, S., Krishnan, P., Krishnan, B., Dryer, S.E., and Hardin, P.E. 2004. Circadian clocks in antennal neurons are necessary and sufficient for olfaction rhythms in Drosophila. Curr. Biol. 14: 638-649.

Tapp, W.N. and Holloway, F.A. 1981. Phase shifting circadian rhythms produces retrograde amnesia. Science 211: 1056-1058.

Tully, T. and Quinn, W.G. 1985. Classical conditioning and retention in normal and mutant Drosophila melanogaster. J. Comp. Physiol. [A] 157: $263-277$.

Valentinuzzi, V.S., Kolker, D.E., Vitaterna, M.H., Ferrari, E., Takahashi, J.S., and Turek, F.W. 2001. Effect of circadian phase on context and cued fear conditioning in C57BL/6J mice. Anim. Learn. Behav. 29: 133-142.

Valentinuzzi, V.S., Menna-Barreto, L., and Xavier, G.F. 2004. Effect of circadian phase on performance of rats in the Morris water maze task. J. Biol. Rhythms 19: 312-324.

van der Horst, G.T., Muijtjens, M., Kobayashi, K., Takano, R., Kanno, S., Takao, M., de Wit, J., Verkerk, A., Eker, A.P., van Leenen, D., et al. 1999. Mammalian Cry1 and Cry2 are essential for maintenance of circadian rhythms. Nature 398: 627-630.

Wagatsuma, A., Sugai, R., Chono, K., Azami, S., Hatakeyama, D., Sadamoto, H., and Itoi, E. 2004. The early snail acquires the learning. Comparison of scores for conditioned taste aversion between morning and afternoon. Acta Biol. Hung. 55: 149-155.

Winocur, G. and Hasher, L. 1999. Aging and time-of-day effects on cognition in rats. Behav. Neurosci. 113: 991-997.

Winocur, G. and Hasher, L. 2004. Age and time-of-day effects on learning and memory in a non-matching-to-sample test. Neurobiol. Aging 25: 1107-1115.

Wolf, F.W., Eddison, M., Lee, S., Cho, W., and Heberlein, U. 2007. GSK-3/ Shaggy regulates olfactory habituation in Drosophila. Proc. Natl. Acad. Sci. 104: 4653-4657.

Yoshii, T., Heshiki, Y., Ibuki-Ishibashi, T., Matsumoto, A., Tanimura, T., and Tomioka, K. 2005. Temperature cycles drive Drosophila circadian oscillation in constant light that otherwise induces behavioural arrhythmicity. Eur. J. Neurosci. 22: 1176-1184.

Zars, T., Fischer, M., Schulz, R., and Heisenberg, M. 2000. Localization of a short-term memory in Drosophila. Science 288: 672-675.

Zhou, X., Yuan, C., and Guo, A. 2005. Drosophila olfactory response rhythms require clock genes but not pigment dispersing factor or lateral neurons. J. Biol. Rhythms 20: 237-244.

Received July 7, 2008; accepted in revised form October 23, 2008. 


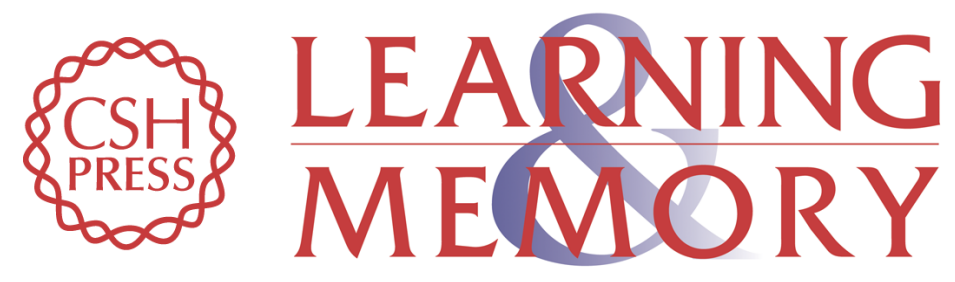

\section{Circadian modulation of short-term memory in Drosophila}

Lisa C. Lyons and Gregg Roman

Learn. Mem. 2009, 16:

Access the most recent version at doi:10.1101//m.1146009

References This article cites 76 articles, 20 of which can be accessed free at: http://learnmem.cshlp.org/content/16/1/19.full.html\#ref-list-1

License

Email Alerting Receive free email alerts when new articles cite this article - sign up in the box at the Service top right corner of the article or click here. 